ESAIM: PROCEEDINGS, October 2011, Vol. 32, p. 76-94

E. Cancès, N. Crouseilles, H. Guillard, B. Nkonga, and E. Sonnendrücker, Editors

\title{
MAGNETIC EQUATIONS WITH FreeFem++: THE GRAD-SHAFRANOV EQUATION \& THE CURRENT HOLE
}

\author{
Erwan Deriaz ${ }^{1}$, Bruno Despres ${ }^{2}$, Gloria FACCANoni ${ }^{3}$, Kirill Pichon GostaF ${ }^{2}$, \\ Lise-MARIE IMBERT-GÉRARD ${ }^{2}$, GEORGES SADAKA ${ }^{4}$ AND REMY SART ${ }^{5}$
}

\begin{abstract}
FreeFem $++[11]$ is a software for the numerical solution of partial differential equations. It is based on finite element method. The FreeFem++ platform aims at facilitating teaching and basic research through prototyping. For the moment this platform is restricted to the numerical simulations of problems which admit a variational formulation. Our goal in this work is to evaluate the FreeFem++ tool on basic magnetic equations arising in Fusion Plasma in the context of the ITER project.

First we consider the Grad-Shafranov equation, which is derived from the static ideal MHD equations assuming axisymetry. Some of the properties of the equation and its analytical solutions are discussed. Second we discretize a reduced resistive MHD model which admits solutions of the Grad-Shafranov equation as stationary solutions. Then the physical stability of these stationary solutions is investigated through numerical experiments and the numerical stability of the algorithm is discussed.
\end{abstract}

\section{INTRODUCTION}

FreeFem++ is a software ${ }^{1}$ which solves partial differential equations numerically with the finite element method. The overall goal of this study is to evaluate the FreeFem++ platform on basic magnetic equations arising in Fusion Plasma.

\subsection{The physical context}

The general context is the ITER ${ }^{2}$ project about thermonuclear fusion in a Tokamak. A nuclear fusion is the process by which multiple nuclei join together to form a heavier nucleus. In order to overcome the electrostatic repulsion between positive ions and to avoid contact between the hot plasma and the material walls, the plasma needs to be confined, for example by magnetic fields. The magnetic confinement structure of a high temperature (107 - 108 mega Kelvin) plasma that we consider in this work is denominated Tokamak, acronym of TOroidalnaya KAmera i MAgnitnaya Katushka from the Russian that means toroidal camera with magnetic

1 Laboratoire de Mécanique, Modélisation et Procédés Propres, 38 rue Frédéric Joliot-Curie 13451 Marseille (France); email: erwan.deriaz@13m. univ-mrs.fr

${ }^{2}$ Laboratoire Jacques-Louis Lions, Université Pierre et Marie Curie, 75252 Paris (France); e-mail: despres@ann.jussieu.fr \& gostaf@ann.jussieu.fr \& imbert@ann.jussieu.fr

${ }^{3}$ Institut de mathématiques de Toulon et du Var, Université du Sud Toulon-Var, 83957 La Garde (France); e-mail: faccanon@univ-tln.fr

${ }^{4}$ Laboratoire Amiénois de Mathématique Fondamentale et Appliquée, Université de Picardie Jules Verne, 33 rue Saint-Leu, 80039 Amiens (France); e-mail: georges.sadaka@u-picardie.fr

5 École Supérieure d'Ingénieurs Léonard de Vinci, 92916 Paris (France); e-mail: remy.sart@devinci.fr

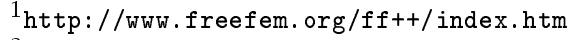

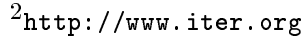

(C) EDP Sciences, SMAI 2011 

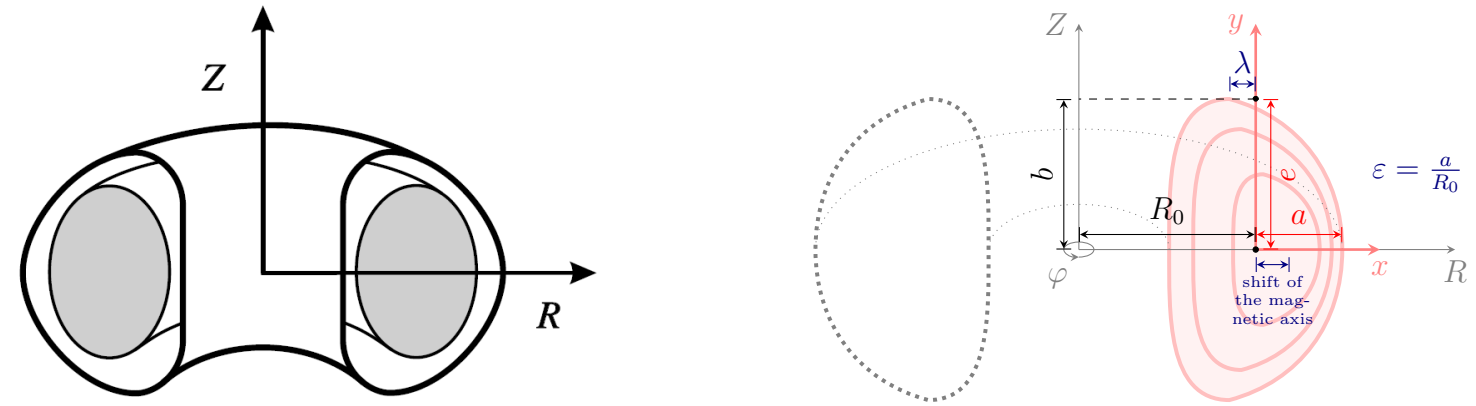

(a) The toroidal vacuum vessel (outer D-shaped(b) The elliptical curve denotes the plasma boundary, whose center is at $R=$ curve) and plasma (shown shaded). $R$ is the radial $R_{0}, Z=0$. The parameter $b$ describes the elongation of the plasma and the and $Z$ the vertical coordinates. triangularity $\lambda$ describes the departure of the plasma boundary from a simple ellipse. (Values of $b=1$ and $\lambda=0$ correspond to a circular plasma boundary). The poloidal shaded domain $\Omega \subset \mathbb{R}^{2}$ will be the computational domain.

Figure 1: Schematic cross-section of a tokamak and definition of various coordinates and parameters. To a good approximation, the tokamak can be regarded as axisymetric about the $Z$-axis, and so the plasma boundary can be described by its cross-sectional shape at one particular toroidal location.

coils (as illustrated schematically in Figure 1a). This design is the one that will be used in ITER. At these temperatures the highly ionized plasma is an excellent electrical conductor, and can be confined and shaped by strong magnetic fields. The main problems to deal with in plasma control are current, position and shape control. Some of these problems have already been subject to thorough research, although it is still possible to improve the behaviour and robustness in the presence of non-linearities.

\subsection{The model}

In this work, the plasma is described by the mono-fluid ideal magnetohydrodynamics equations under the assumption of axisymetry. Plasma floats in a somewhat D-shaped configuration surrounded by a conducting wall. Between the hot plasma and the wall is a vacuum such that the plasma has to be kept in place with external magnetic fields. Superconducting magnets produce a large toroidal (i.e. in the $\phi$-direction) magnetic field that, combined with poloidal (i.e. in the cross-sectional plane) electric currents inside the plasma, produce an inward Lorentz force counteracting the pressure force. An additional toroidal electric current produces a poloidal magnetic field also constituting an inward Lorentz force. The position and shape of the plasma boundary can be described in terms of a set of geometrical parameters such as vertical position and elongation, illustrated in Figure 1b in which a cross-section of an axisymmetric toroidal tokamak is shown. The axis of symmetry is the $Z$-axis and the ignorable angle is $\phi . R$ is a radial coordinate, $R_{0}$ and $a$ are called the major axis and minor axis of the tokamak, $e=b / a$ is the ellipticity, $\varepsilon=a / R_{0}$ is the inverse aspect ratio and $\lambda$ the triangularity. Supposing invariance along the toroidal direction $\phi$, the dimensionless non-linear equations write in the form given in [4-6]

$$
\left\{\begin{array}{l}
\partial_{t} \psi=(1+\varepsilon x)[\psi, \varphi]+\eta\left(J-J_{c}\right), \\
\partial_{t} \omega=2 \varepsilon \omega \frac{\partial \varphi}{\partial y}+(1+\varepsilon x)[\omega, \varphi]+\frac{1}{1+\varepsilon x}[\psi, J]+\nu \Delta_{\perp} \omega, \\
J=\Delta^{*} \psi \\
\omega=\Delta_{\perp} \varphi
\end{array}\right.
$$


The unknowns are the magnetic flux $\psi$ (that is $\mathbf{B}=\nabla \times \psi$ is the magnetic field), the velocity potential $\varphi$, the toroidal current density $J$ and the vorticity $\omega$. The Poisson brackets are denoted $[\cdot, \cdot]$ with properties

$$
[a, b]=\frac{\partial a}{\partial x} \frac{\partial b}{\partial y}-\frac{\partial a}{\partial y} \frac{\partial b}{\partial x}=\nabla \cdot(a(\nabla \times b))=-[b, a], \quad[a,[b, c]]=-[b,[c, a]]-[c,[a, b]] \quad \text { and } \quad[a b, c]=a[b, c]+b[a, c] .
$$

The operator $\Delta^{*}$ is the Grad-Shafranov operator defined in section 2. The operator $\Delta_{\perp}$ is the laplacian restricted to the poloidal section. The parameter $\eta$ stands for the resistivity, $\nu$ for the viscosity. We implicitly assume a uniform density. The source term $J_{c}$ represents a non-ohmic driven current density that sets a constant profile likely to be perturbed with fluctuations. It is natural to impose homogeneous Dirichlet boundary conditions on the exterior frontier

$$
\left.u\right|_{\partial \Omega}=\left.\varphi\right|_{\partial \Omega}=\left.\psi\right|_{\partial \Omega}=0
$$

\subsection{Outline}

Non-linear MHD simulations typically start from an equilibrium: we study in section 2 the stationary solution of the so-called Grad-Shafranov equation

$$
-\Delta^{*} \psi=J_{c},\left.\quad \psi\right|_{\partial \Omega}=0
$$

We present first how to compute the solution with FreeFem++ in the case of the Soloviev equilibrium which corresponds to a $J_{c}$ independent of $\psi$. Then we generalize the algorithm to the case of a polynomial (in $\psi$ ) non ohmic current. Then in section 3 we study the stability of the stationary solution in the simplified cylindrical case (i.e. when $\varepsilon=0$ ) via the numerical solution of

$$
\left\{\begin{array}{l}
\partial_{t} \psi=[\psi, \varphi]+\eta\left(J-J_{c}\right) \\
\partial_{t} \omega=[\omega, \varphi]+[\psi, J]+\nu \Delta \omega \\
J=\Delta \psi \\
\omega=\Delta \varphi
\end{array}\right.
$$

We introduce some schemes and the conclusion will be that the stationary solution that we consider is unstable: this is what is called the current hole [6].

\section{THE EQUILIBRIUM: STATIONARY SOLUTIONS}

The equilibrium refers to the stationary solution of (3).

\subsection{The Grad-Shafranov operator}

In this part we study the Grad-Shafranov equation (2) $[1,13]$ that models a plasma equilibrium where the pressure gradient balances the Lorentz force, Ampere's law and the fact that the magnetic field $\mathbf{B}$ is divergencefree (see for example $[3,6,8]$ ). The function $\psi$ is the magnetic flux and the plasma boundary corresponds to a surface of constant $\psi$. The Grad-Shafranov operator $\Delta_{c}^{*}$ is a second-order elliptic operator (the coordinates $R$ and $Z$ are defined in Figure 1a)

$$
\Delta_{c}^{*} \psi:=R^{2} \operatorname{div}_{c}\left(\frac{1}{R^{2}} \nabla_{c} \psi\right)
$$

where $\operatorname{div}_{c}$ and $\nabla_{c}$ respectively stands for the divergence and the gradient in the poloidal section for cylindrical coordinates, namely $\nabla_{c} A=\left(\partial_{R} A, \partial_{Z} A\right)^{T}$ and $\operatorname{div}_{c} \mathbf{A}=R^{-1} \partial_{R}\left(R A_{R}\right)+\partial_{Z} A_{Z}$. The function $J_{c}$ specifies the 
plasma current density $J_{c}(R, \psi)=R^{2} p^{\prime}(\psi)+F(\psi) F^{\prime}(\psi)$, where $p$ is the pressure, and $F=R B_{\phi}$, with $B_{\phi}$ the toroidal component of the magnetic field. Then the equation (2) writes

$$
-R \frac{\partial}{\partial R}\left(\frac{1}{R} \frac{\partial \psi}{\partial R}\right)-\frac{\partial^{2} \psi}{\partial Z^{2}}=R^{2} \frac{\mathrm{d} p}{\mathrm{~d} \psi}+F \frac{\mathrm{d} F}{\mathrm{~d} \psi}
$$

Due to the non-linear nature of the Grad-Shafranov equation, a general analytic solution is not possible. However, for a given RHS, the Grad-Shafranov equation can be solved numerically, with boundary conditions determined by currents showing in the external control coils which surround the vacuum vessel. A considerable complication is that the plasma cross-section (i.e. the value of $\psi$ along a curve), in general, is also unknown since it represents the interface between the plasma and the external vacuum region which is determined by another non-linear problem, viz. the external free-boundary problem with given currents in external coils. Here, we will assume that this problem is solved separately so that we prescribe a desired shape of the plasma cross-section.

Definition 2.1 (Soloviev equilibrium). The Grad-Shafranov equation (4), together with the conditions $p^{\prime}(\psi)=$ $\alpha=$ cst and $F(\psi) F^{\prime}(\psi)=\beta=$ cst, is the so-called Soloviev equilibrium. This solution is equivalent to Hill's spherical vortex solution from fluid mechanics, with $\psi$ representing the Stokes stream function.

To describe the poloidal plane of a torus, let us define the corresponding dimensionless Cartesian coordinates by the relations (see Figure $1 \mathrm{~b}) R=R_{0}+a x=R_{0}(1+\varepsilon x)$ and $Z=a y$. Then the equation (4), together the Soloviev equilibrium, can be fully expressed with the $(x, y)$-coordinates as

$$
-\frac{1}{a^{2}}\left((1+\varepsilon x) \nabla \cdot\left(\frac{\nabla \psi}{1+\varepsilon x}\right)\right)=\left(\alpha R_{0}^{2}(1+\varepsilon x)^{2}+\beta\right),
$$

where the operator $\nabla$. and $\nabla$ here stand for the classical divergence and gradient in the Cartesian coordinates.

\subsection{Weak formulation}

The convenient mathematical formulation of the problem is the following. We multiply the equation (5) by $a^{2} \varphi /(1+\varepsilon x)$ where the test function is $\varphi$ and we integrate by parts on the domain $\Omega$. Using the homogeneous boundary condition we readily obtain the formulation "find $\psi \in V$ such that $a(\psi, \varphi)=\ell(\varphi)$ for all $\varphi \in V$ " where the bilinear form and the linear right hand side are

$$
a(\psi, \varphi)=\int_{\Omega} \frac{\nabla \psi \cdot \nabla \varphi}{1+\varepsilon x} \mathrm{~d} \Omega, \quad \ell(\varphi)=\int_{\Omega} a^{2}\left(\alpha R_{0}^{2}(1+\varepsilon x)+\frac{\beta}{1+\varepsilon x}\right) \varphi \mathrm{d} \Omega
$$

and the convenient functional space is $V=H_{0}^{1}(\Omega)$. This weak formulation fits within the frame of the LaxMilgram theorem since the bilinear form $a$ is symmetric, continuous and coercive. The linear form $\ell$ is continuous. This ensures the existence and uniqueness of the solution $\psi$. Other boundary conditions would lead to a different bilinear form $a$, a different linear form $\ell$ or a slightly different space $V$ but would not compromise the conclusion of Lax-Milgram theorem.

\subsection{Computational examples}

We use the finite element method with linear triangular elements in order to compute the numerical solutions, as described in the FreeFem++ manual [11]. In order to perform a validation of the code, we present here a set of computational examples for some analytical solutions that can be found in the literature. A first example permits to describe the structure of the code and to compare results obtained with a boundary elements method. In a second example a very good convergence is observed. The following examples focus on the handling of different types of right hand side depending on $\psi$. In the third example, the right hand side is linear in $\psi$. In the fourth example, non-linear right hand sides are considered, so that a linearisation scheme has to be chosen. 


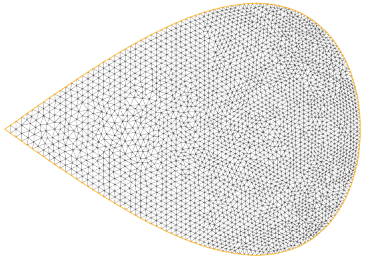

(a) $N_{\partial \Omega}=200$

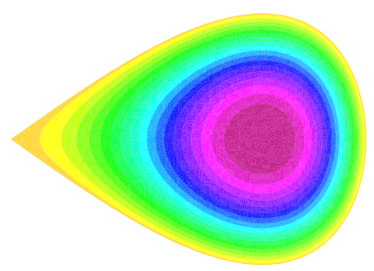

(b) $\psi_{\text {ex }}$

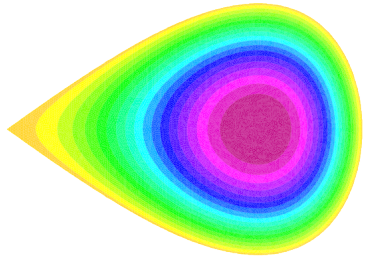

(c) $\psi$

Figure 2: Example I: comparison of the exact magnetic flux $\psi_{\text {ex }}$ and the numerical magnetic flux $\psi$ with $\mathbb{P}^{1}$ finite elements. We also plot the mesh.

\subsubsection{A first example}

The domain $\Omega$ is defined by its frontier $\partial \Omega=\left\{(R, Z) \mid R=R_{0} \sqrt{1+\frac{2 a \cos \alpha}{R_{0}}}, Z=a R_{0} \sin \alpha, \alpha=0: 2 \pi\right\}$. The exact solution is given [2]:

$$
\psi(R, Z)=\frac{f_{0} R_{0}^{2}}{2}\left(a^{2}-Z^{2}-\frac{\left(R^{2}-R_{0}^{2}\right)^{2}}{4 R_{0}^{2}}\right) .
$$

For the parameter choice $a=0.5, f_{0}=1$ and $R_{0}=1$, the numerical results are displayed in Figure 2. A comparison is possible with [2] where the numerical solution is obtained with the Boundary Element Method. To show how to compute the solution with FreeFem++ and estimate the error, let us go through all the steps one by one (for more details see [11]).

- We declare the variables.

int $\mathrm{n}=200 ; / / \mathrm{n}$ is the number of the points in the border $\partial \Omega$

real L2error;

- The right hand side $g$, the boundary condition $b$ and the exact solution $\psi_{\text {ex }}$ are defined analytically (in FreeFem++ variables must be noted $x$ and $y$ ).

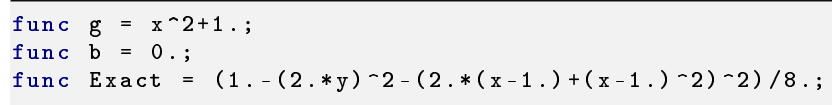

- The boundary is described analytically by a parametric equation for $x$ and for $y$. The mesh of the domain is automatically generated by using $n$ points on the border. The domain is assumed to be on the left side of the boundary which is implicitly oriented by the parametrization. As $\Omega$ is not a polygonal domain, a "skin" remains between the exact domain and its approximation. However, we notice that all corners of the triangulation lies in $\partial \Omega$. The visualization of the triangulation is done and saved in a postscript file.

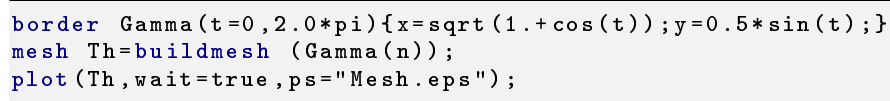

- The finite element space is a space of polynomial functions on elements, triangles here, with certain matching properties at edges, vertices etc. Here we define the space $\mathbb{P}^{1}$ of continuous piecewise linear functions which are equal to 1 on one vertex of the triangulation and 0 on all others. We declare that $\psi, \psi_{\mathrm{ex}}, \varphi$ and $\left|\psi-\psi_{\mathrm{ex}}\right|$ are approximated as continuous piecewise linear functions. 


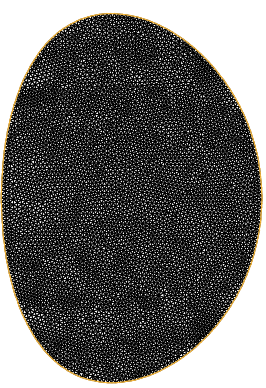

(a) $N_{\partial \Omega}=128$

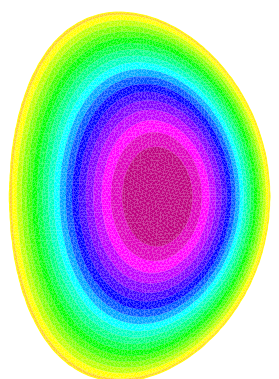

(b) $\psi_{\mathrm{ex}}$

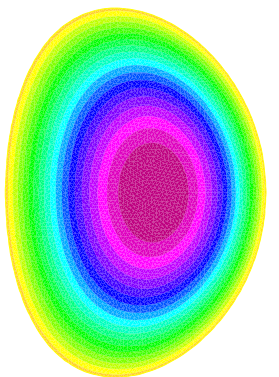

(c) $\psi$

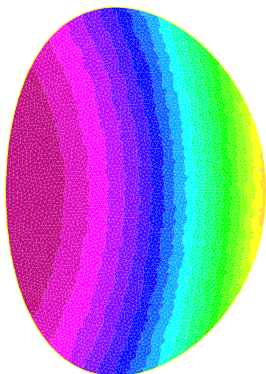

(d) $\frac{\partial \psi_{\text {ex }}}{\partial x}$

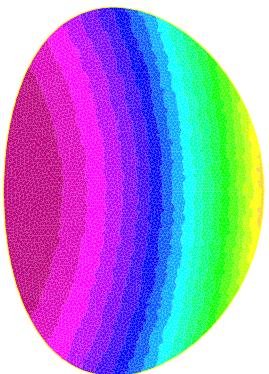

(e) $\frac{\partial \psi}{\partial x}$

Figure 3: Second example: comparison of the exact magnetic flux $\psi_{\text {ex }}$ and the numerical magnetic flux $\psi$. We also plot the mesh and the $x$-derivative.

- The visualization of the exact solution is done and saved in a postscript file.

psiExact $=$ Exact;

plot (psiExact, wait=true, fill=true, value=true,ps="Exact .eps");

- We define the bilinear form of Grad-Shafranov equation and its Dirichlet boundary conditions. In FreeFem++ there is no need to distinguish the bilinear form from the linear form. As long as the terms are inside different integrals, FreeFem++ interpreter finds out which one is the bilinear form by checking where both terms $\psi$ and $\varphi$ are present. The problem is solved, the visualization of the approximation is done and saved in a postscript file.

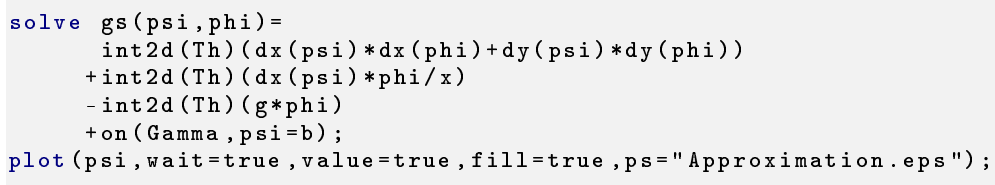

- The error $E(\psi)=\left\|\psi-\psi_{\mathrm{ex}}\right\|_{L^{2}} /\left\|\psi_{\mathrm{ex}}\right\|_{L^{2}}$ is computed and written in the console.

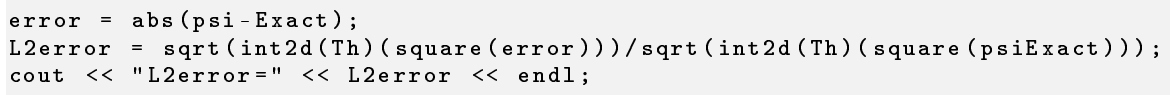

- Results are compared with the exact solution and represented in terms of contours in Figure 2.

\subsubsection{Second example: Soloviev Equilibrium with triangularity parameter}

In this example we test the solver over a class of analytical solutions which can be written in Cartesian coordinates as

$$
\psi(x, y)=1-\left(x-\frac{\varepsilon}{2}\left(1-x^{2}\right)\right)^{2}-\left(\left(1-\frac{\varepsilon^{2}}{4}\right)(1+\varepsilon x)^{2}+\lambda x\left(1+\frac{\varepsilon}{2} x\right)\right)\left(\frac{a}{b} y\right)^{2}
$$

where $\lambda$ stands for the triangularity parameter. A straightforward computation shows that this solution corresponds to a Soloviev equilibrium, i.e.

$$
-\Delta^{*} \psi=\alpha\left(R_{0}(1+\varepsilon x)\right)^{2}+\beta \quad \text { with } \quad \alpha=\frac{4\left(a^{2}+b^{2}\right) \varepsilon+a^{2}\left(2 \lambda-\varepsilon^{3}\right)}{2 R_{0}^{2} \varepsilon a^{2} b^{2}} \quad \text { and } \quad \beta=-\frac{\lambda}{b^{2} \varepsilon} .
$$


We remark that $\psi=0$ going through $( \pm 1,0)$ and $\left(0, \pm \frac{b}{a}\right)$; the shift of the magnetic axis is then given by $\left(\sqrt{1+\varepsilon^{2}}-1\right) \varepsilon$. For the need of further comparisons with [6], in our tests we use the following parameters: the ellipticity $\varepsilon=3 / 10$, the triangularity $\lambda=0$, the major axis $R_{0}=5 / 3$, the minor axis $a=1 / 2$ and the elongation $b=7 / 10$, which implies $\alpha \approx 4.35$ and $\beta=0$. The geometry is the $D-$ shaped configuration defined by $\partial \Omega=\left\{(x, y) \in \mathbb{R}^{2} \mid \psi(x, y)=0\right\}$, i.e.

$$
y= \pm \frac{b}{a} \sqrt{\frac{1-\left(x-\frac{\varepsilon}{2}\left(1-x^{2}\right)\right)^{2}}{\left(1-\frac{\varepsilon^{2}}{4}\right)(1+\varepsilon x)^{2}+\lambda x\left(1+\frac{\varepsilon}{2} x\right)}}, \quad x \in[-1 ; 1]
$$

In Figure 3 we draw the mesh generated by FreeFem++ with $N_{\partial \Omega}=128$ elements on the border, the magnetic flux $\psi_{\text {ex }}$ and the approximated magnetic flux $\psi$ computed with $\mathbb{P}^{1}$ finite elements and the exact and approximated derivatives $\partial_{x} \psi_{\text {ex }}$ and $\partial_{x} \psi$. For a given function $\xi$ and its numerical approximation $\xi_{\text {ex }}$, the error $E(\xi)$ is defined as the relative difference in the $L^{2}$ norm

$$
E(\xi)=\frac{\left\|\xi-\xi_{\mathrm{ex}}\right\|_{L^{2}}}{\left\|\xi_{\mathrm{ex}}\right\|_{L^{2}}}=\sqrt{\frac{\int_{\Omega}\left(\xi-\xi_{\mathrm{ex}}\right)^{2} \mathrm{~d} \Omega}{\int_{\Omega}\left(\xi_{\mathrm{ex}}\right)^{2} \mathrm{~d} \Omega}}
$$

We obtain the following table of convergence

\begin{tabular}{rll}
\hline$N_{\partial \Omega}$ & \multicolumn{1}{c}{$E(\psi)$} & \multicolumn{1}{c}{$E(\partial \psi / \partial x)$} \\
\hline 22 & 0.0630042 & 0.0467509 \\
38 & 0.0326535 & 0.0272896 \\
64 & 0.00706633 & 0.0109076 \\
128 & 0.00242758 & 0.00517435 \\
255 & 0.000728738 & 0.00240365 \\
517 & 0.000269108 & 0.00127819 \\
1067 & 0.000009828 & 0.000653738 \\
\hline
\end{tabular}

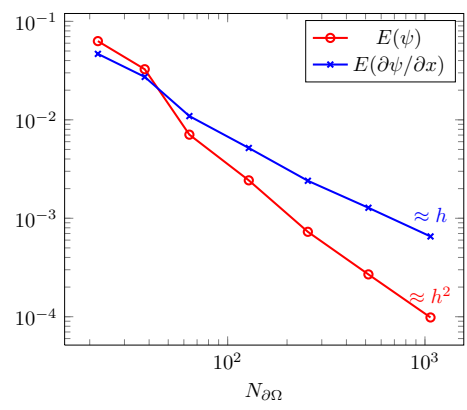

Numerical results match perfectly the theoretical expectations: $\mathbb{P}^{1}$ elements provide a second convergence rate for the magnetic flux, while gradients are approximated at first order.

\subsubsection{Third example: Linear RHS}

We consider here a first example of RHS without Soloviev equilibrium:

$$
\frac{1}{R} \frac{\partial \psi}{\partial R}-\frac{\partial^{2} \psi}{\partial R^{2}}-\frac{\partial^{2} \psi}{\partial Z^{2}}=\left(R^{2}+1\right) \psi
$$

Straightforward computation shows that the analytical solution is $\psi(R, Z)=\cos \left(\frac{R^{2}}{2}\right) \cos (Z)-\frac{1}{2}$. The computational domain $\Omega$ is

$$
\partial \Omega=\left\{(R, Z) \mid R \in\left[\frac{2}{3} \sqrt{3 \pi}, \frac{2}{3} \sqrt{6 \pi}\right], Z=\arccos \left(\frac{1}{2 \cos \frac{R^{2}}{2}}\right) \cup Z=2 \pi-\arccos \left(\frac{1}{2 \cos \frac{R^{2}}{2}}\right)\right\}
$$

Figure 4 shows the exact and the approximated solutions represented in terms of contours obtained by FreeFem++ with $\mathbb{P}^{1}$ finite elements: the numerical solution agrees very well with the analytical one. 


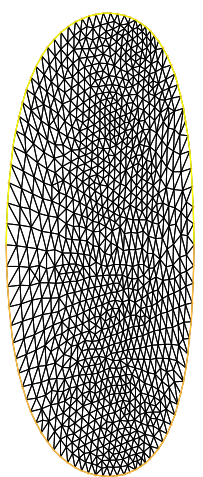

(a) Mesh

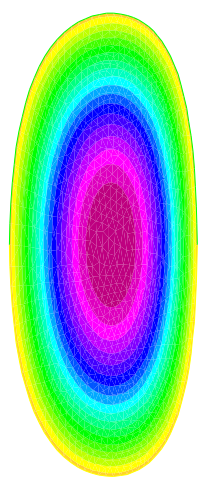

(b) $\psi_{\text {ex }}$

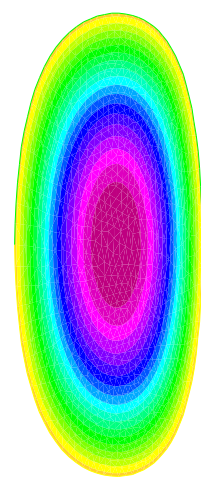

(c) $\psi$

Figure 4: Third example: comparison of the exact solution $\psi_{\text {ex }}$ and the numerical solution $\psi$. We also plot the mesh.

\subsubsection{Fourth example: Polynomial non-linear RHS}

In this section we consider a non-linear right hand side for the Grad-Shafranov equation. There is no result concerning uniqueness of the solution, but we would still like to adapt the previous method by defining an iterative sequence. In what follows we study the convergence of the algorithm to a know analytical solution.

Consider a general problem described by

$$
-\Delta_{c}^{*} \psi=G(\psi)
$$

where the non-linear function $G$ can be written as $\psi G_{1}(\psi)+G_{2}(\psi)$. A Picard's iteration (8) starts from any initial function, then each calculus step solves a linear problem which depends on the previous step's result. Each step is performed by solving the same linear problem corresponding to the example 3 , so that the basic method is kept unchanged. The issue is then to choose how to define this linear formulation. To obtain the desired linear right hand side with respect to $\psi$, the functions $G_{1}$ and $G_{2}$ has to depend only on the result of the previous step, denoted $\psi_{n}$. From then on we consider the algorithm

$$
\left\{\begin{array}{l}
\psi_{0} \text { given, } \\
-\Delta_{c}^{*} \psi_{n+1}=\psi_{n+1} G_{1}\left(\psi_{n}\right)+G_{2}\left(\psi_{n}\right),
\end{array}\right.
$$

and the iteration is stopped when $\mathcal{N}\left(\psi_{n+1}-\psi_{n}\right)<$ tol where $\mathcal{N}$ is a norm and tol a prescribed tolerance. In fact each choice of $G_{1}$ and $G_{2}$ gives a new scheme.

Here we detail the case $G(\psi)=\psi^{2}\left(k_{1}+k_{2} R^{2} \psi\right)$, together with Dirichlet boundary conditions, on the domain $\Omega$ defined in poloidal coordinates by $R \in] 0,1[$ and $z \in]-1,1\left[\right.$. When $k_{2}=2 a\left(k_{1}-9 a\right)$, an analytic solution of this equation is given [12] by $\psi_{e x}=-6 /\left(9 a R^{2}+k_{1}\left(z+c_{1}\right)^{2}\right)$. The variational formulation reads

$$
\int_{\Omega}\left(\frac{\partial \psi}{\partial R} \frac{\partial \varphi}{\partial R}+\frac{\partial \psi}{\partial z} \frac{\partial \varphi}{\partial z}+\frac{1}{R} \frac{\partial \psi}{\partial R} \varphi\right)=\int_{\Omega}\left(k_{1} \psi^{2} \varphi+k_{2} R^{2} \psi^{3} \varphi\right)+\text { boundary terms }
$$

so that the point is now to choose the implicit and explicit parts of the second integral. As explained earlier, the aim is to obtain a linear term in $\psi_{n+1}$ plus a term only depending on $\psi_{n}$. Let $(\beta, \delta)$ be parameters and let 
us write the second term

$$
\int_{\Omega}\left(k_{1}\left(\psi_{k}\right)^{2-\beta}\left(\psi_{k+1}\right)^{\beta} \varphi+k_{2} R^{2}\left(\psi_{k}\right)^{3-\delta}\left(\psi_{k+1}\right)^{\delta} \varphi\right) .
$$

In order to get the desired form, the parameters $(\beta, \delta)$ have to belong to $\{0,1\}^{2}$ : it provides at most linear terms in the unknown $\psi_{k+1}$. In our test the starting point for the iterative method is a perturbation of the exact solution: $\psi_{0}=\psi_{\text {ex }}(1+R(R-1)(z+1)(z-1))$; the parameters are $k_{1}=0.1, a=0.1$ and $c_{1}=2$, and the domain studied is the square $\Omega=\{(R, z) \in[0,1] \times[-1,1]\}$. Here is the description of the different schemes:

\begin{tabular}{ccccc}
\hline Scheme & $(1)$ & $(2)$ & $(3)$ & $(4)$ \\
\hline$\beta$ & 0 & 0 & 1 & 1 \\
$\delta$ & 0 & 1 & 0 & 1 \\
\hline
\end{tabular}

We tested this four schemes with $\mathbb{P}^{1}$ and $\mathbb{P}^{2}$ types finite elements and tol $=10^{-5 / 2}$ to stop the iterations. It is obvious that the behavior of the scheme is governed by the higher order polynomial term (described by $\delta$ ): an implicit term has to be chosen there to allow any hope of convergence toward the analytical solution. We define the relative error in $\mathcal{N}$ norm as $E(\psi)=\mathcal{N}\left(\psi-\psi_{e x}\right) / \mathcal{N}\left(\psi_{e x}\right)$. Using the $\mathbb{P}^{1}$ elements, the scheme (2) shows a convergence order of 1.712 in $\mathbb{L}^{2}$ norm, 1.034 in $\mathbb{H}^{1}$ norm. The scheme (3) a convergence order of 1.608 in $\mathbb{L}^{2}$ norm, 1.031 in $\mathbb{H}^{1}$ norm and the scheme (4) a convergence order of 1.764 in $\mathbb{L}^{2}$ norm, 1.036 in $\mathbb{H}^{1}$ norm. Another kind of behavior is observed for the scheme (1): it seems that the computation converges, but to another solution. In fact, as the problem is non linear, the equation may have several solutions. Here we underline the fact that the schemes (2) and (4) which seem to converge to the given analytical solution, both make implicit the term of higher degree in the polynomial right hand side. Both of them satisfy $\delta=1$. More research is needed to obtain more definitive conclusions for this problem. In particular one may think of studying the influence on the non linearity and of the amplitude of the initial perturbation.

\section{The Current Hole: unstable equilibrium}

This part is devoted to the implementation of the time-dependent problem (3). For the need of further comparisons with analytical work, we simplify the problem and choose the planar cylindrical geometry: that is $\varepsilon=0$ (it is not a restriction). In this case $\Delta^{*}=\Delta_{\perp}=\Delta$. Thus the model reads $(x \in \Omega, t \in[0, T])$

$$
\left\{\begin{array}{l}
\partial_{t} \psi=[\psi, \varphi]+\eta\left(J-J_{c}\right), \\
\partial_{t} \omega=[\omega, \varphi]+[\psi, J]+\nu \Delta \omega, \\
J=\Delta \psi \\
\omega=\Delta \varphi
\end{array}\right.
$$

This is a non linear and non stationary system of partial differential equations. As the initial conditions for the time-dependent simulations, we use the solution of the Grad-Shafranov equilibrium based on current profile. The velocity and vorticity are set to zero. Thus at $t=0$ we set

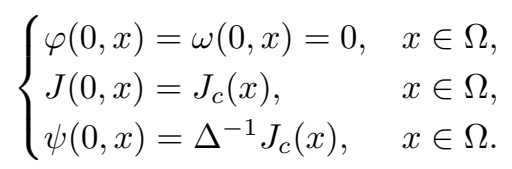

To close the system we consider as before the homogeneous Dirichlet boundary conditions for all variables

$$
\varphi(t, x)=\omega(t, x)=\psi(t, x)=J(t, x)=0, \quad x \in \partial \Omega, t \in[0, T] .
$$


The system (12) verifies some conservation properties.

Proposition 3.1 (Energy Conservation). Assume $\eta=\nu=0$. Then regular solutions of (12) satisfy

$$
\frac{1}{2} \frac{\mathrm{d}}{\mathrm{d} t} \int_{\Omega}|\nabla \psi|^{2}+|\nabla \varphi|^{2} \mathrm{~d} \mathbf{x}=0
$$

Remark 3.2. Notice that $\int_{\Omega}\left|\nabla \psi_{n+1}\right|^{2}+\left|\nabla \varphi_{n+1}\right|^{2} \mathrm{~d} \mathbf{x}=\int_{\Omega}|\mathbf{B}|^{2}+|\mathbf{u}|^{2} \mathrm{~d} \mathbf{x}$ is the total energy. If one assumes that $J_{c}=0$ but $\eta>0$ and $\nu>0$, then one gets

$$
\frac{1}{2} \frac{\mathrm{d}}{\mathrm{d} t} \int_{\Omega}|\nabla \psi|^{2}+|\nabla \varphi|^{2} \mathrm{~d} \mathbf{x} \leq 0
$$

Proof. With the assumption $\eta=\nu=0$, and knowing that $J=\Delta \psi$ and $\omega=\Delta \varphi$, the model (12) writes

$$
\left\{\begin{array}{l}
\partial_{t} \psi=[\psi, \varphi] \\
\partial_{t}(\Delta \varphi)=[\Delta \varphi, \varphi]+[\psi, \Delta \psi]
\end{array}\right.
$$

Multiplying (13a) by $\Delta \psi$ and (13b) by $\varphi$ and integrating over $\Omega$, we get

$$
\begin{gathered}
\frac{\mathrm{d}}{\mathrm{d} t} \int_{\Omega}|\nabla \psi|^{2} \mathrm{~d} \mathbf{x}+\int_{\Omega}[\psi, \varphi] \Delta \psi \mathrm{d} \mathbf{x}=0, \\
\frac{\mathrm{d}}{\mathrm{d} t} \int_{\Omega}|\nabla \varphi|^{2} \mathrm{~d} \mathbf{x}+\int_{\Omega}[\Delta \varphi, \varphi] \varphi \mathrm{d} \mathbf{x}+\int_{\Omega}[\psi, \Delta \psi] \varphi \mathrm{d} \mathbf{x}=0 .
\end{gathered}
$$

The energy identity is finally obtained using the following property of the Poisson brackets

$$
\int_{\Omega}[a, b] c \mathrm{~d} \mathbf{x}=\int_{\Omega} a[b, c] \mathrm{d} \mathbf{x}+\int_{\partial \Omega} a c(\nabla \times b \cdot \mathbf{n}) \mathrm{d} \sigma
$$

where $\mathbf{n}$ is the outgoing normal to the surface $\mathrm{d} \sigma$, together with the boundary conditions $\psi=\varphi=0$ on $\partial \Omega$, from (1).

Proposition 3.3 (Magnetic Helicity Conservation). With the same hypotheses as in proposition 3.1, one has

$$
\frac{\mathrm{d}}{\mathrm{d} t} \int_{\Omega} \psi \mathrm{d} \mathbf{x}=0
$$

Proof. See [3, page 21]. Since the right hand side of the $\psi$ equation can be written is divergent form, this is immediate.

Proposition 3.4 (Cross-Helicity Conservation). With the same hypotheses as in proposition 3.1, one has

$$
\frac{\mathrm{d}}{\mathrm{d} t} \int_{\Omega} \nabla \varphi \cdot \nabla \psi \mathrm{d} \mathbf{x}=-\frac{\mathrm{d}}{\mathrm{d} t} \int_{\Omega} \omega \psi \mathrm{d} \mathbf{x}=0 .
$$

Proof. See [3, page 21].

Consistently with [6], we will choose in our numerical tests the following radial profile for the source term

$$
J_{c}=\frac{\left(1-R^{4}\right)}{5}-0.266\left(1-R^{2}\right)^{8}
$$

with $R^{2}=x_{1}^{2}+x_{2}^{2}$ for $\mathbf{x} \in \Omega=\mathcal{C}(0,1)$, and the viscosity parameters $\eta=1 \cdot 10^{-5}$ and $\nu=1 \cdot 10^{-6}$. 


\subsection{Von Neumann stability analysis}

In the following paragraph, we show that it is possible to solve the MHD reduced system (12) numerically using an explicit time scheme for all the non-linear terms and that this scheme is stable under a CFL condition $\delta t \leq C h$ where $h$ is the minimal space step. Let $X=(\delta \psi, \delta \varphi, \delta J, \delta \omega)^{T}$ and $\partial_{t} X=F(X)$. In order to apply the von Neumann stability analysis, we linearise the system of equations. Taking $X=\bar{X}+X^{\prime}$, with $\partial_{t} \bar{X}=F(\bar{X})$ and $\left\|X^{\prime}\right\| \ll\|\bar{X}\|$, we have

$$
\partial_{t} X=\partial_{t} \bar{X}+\partial_{t} X^{\prime}=F\left(\bar{X}+X^{\prime}\right)=F(\bar{X})+\nabla F(\bar{X}) \cdot X^{\prime}+o\left(X^{\prime}\right)
$$

so the linearization [10] provides $\partial_{t} X^{\prime}=\nabla F(\bar{X}) \cdot X^{\prime}$. In our case, this corresponds to

$$
\left\{\begin{array}{l}
\partial_{t} \psi^{\prime}=\left[\bar{\psi}, \varphi^{\prime}\right]+\left[\psi^{\prime}, \bar{\varphi}\right]+\eta \Delta \psi^{\prime} \\
\partial_{t} \omega^{\prime}=\left[\bar{\omega}, \varphi^{\prime}\right]+\left[\omega^{\prime}, \bar{\varphi}\right]+\left[\bar{\psi}, J^{\prime}\right]+\left[\psi^{\prime}, \bar{J}\right]+\nu \Delta \omega^{\prime} \\
J^{\prime}=\Delta \psi^{\prime} \\
\omega^{\prime}=\Delta \varphi^{\prime}
\end{array}\right.
$$

We remark that the diffusive terms $\eta \Delta \psi^{\prime}$ and $\nu \Delta \omega^{\prime}$ integrated reasonably well (i.e. in an implicit scheme or explicitly under the condition $\delta t \leq h^{2} / \eta$ and $\left.\delta t \leq h^{2} / \nu\right)$ smooth out the solution. Then the interesting case is when $\eta$ and $\nu$ are small, so let us make the assumption that $\eta$ and $\nu$ vanish, and see what happens regarding the linear stability of the equations.

We assume that $X^{\prime}=e^{i\left(\xi_{1} x_{1}+\xi_{2} x_{2}\right)} X_{0}$, then $J^{\prime}=-|\xi|^{2} \psi^{\prime}, \omega^{\prime}=-|\xi|^{2} \varphi^{\prime}$ and, from (14a) and (14b),

$$
\left\{\begin{array}{l}
\partial_{t} \psi^{\prime}=\left[\bar{\psi}, \varphi^{\prime}\right]+\left[\psi^{\prime}, \bar{\varphi}\right] \\
\partial_{t} \varphi^{\prime}=-\frac{1}{|\xi|^{2}}\left[\bar{\omega}, \varphi^{\prime}\right]+\left[\varphi^{\prime}, \bar{\varphi}\right]+\left[\bar{\psi}, \psi^{\prime}\right]-\frac{1}{|\xi|^{2}}\left[\psi^{\prime}, \bar{J}\right] .
\end{array}\right.
$$

As we study the numerical instabilities for large values of $|\xi|$, we can neglect the two terms multiplied by $1 /|\xi|^{2}$. Then knowing that $[a, b]=-[b, a]$ we write:

$$
\left\{\begin{array}{l}
\partial_{t} \psi^{\prime}=\left[\bar{\psi}, \varphi^{\prime}\right]-\left[\bar{\varphi}, \psi^{\prime}\right] \\
\partial_{t} \varphi^{\prime}=-\left[\bar{\varphi}, \varphi^{\prime}\right]+\left[\bar{\psi}, \psi^{\prime}\right]
\end{array}\right.
$$

i.e.

$$
\frac{\partial}{\partial t}\left(\begin{array}{l}
\psi^{\prime} \\
\varphi^{\prime}
\end{array}\right)=\mathbb{M}\left(\begin{array}{c}
\psi^{\prime} \\
\varphi^{\prime}
\end{array}\right) \quad \text { with } \quad \mathbb{M}(\cdot):=\left(\begin{array}{cc}
-[\bar{\varphi}, \cdot] & {[\bar{\psi}, \cdot]} \\
{[\bar{\psi}, \cdot]} & -[\bar{\varphi}, \cdot]
\end{array}\right)
$$

Putting $\xi=|\xi|(\cos \theta, \sin \theta)$, the operator-matrix $\mathbb{M}$ is symmetric and can be put under the form:

$$
\mathbb{M}=i|\xi| \mathbb{Q}_{\theta}\left(\begin{array}{cc}
\mu_{\theta}^{(1)} & 0 \\
0 & \mu_{\theta}^{(2)}
\end{array}\right) \mathbb{Q}_{\theta}^{-1}
$$

with $\mathbb{Q}_{\theta}$ independent of $|\xi|$ and $0 \leq \mu_{\theta}^{(1)}, \mu_{\theta}^{(2)} \leq\|\nabla \varphi\|_{L^{\infty}}+\|\nabla \psi\|_{L^{\infty}}$.

As a conclusion, the reduced MHD model (12) is equivalent to a transport equation regarding the local numerical stability. Hence, high order explicit schemes (see Figure 5 for the domains of stability) are stable under the CFL condition $\delta t \leq C h / \mu$ with $C \approx 1$ a constant depending on the scheme and $\mu=\|\nabla \varphi\|_{L^{\infty}}+\|\nabla \times \psi\|_{L^{\infty}}$.

\subsection{Numerical Discretization}

The von Neumann stability analysis shows that, although possible, an explicit time discretization of (12) leads to a restrictive CFL condition. Therefore, in addition to the Adams-Bashforth order two scheme treated 

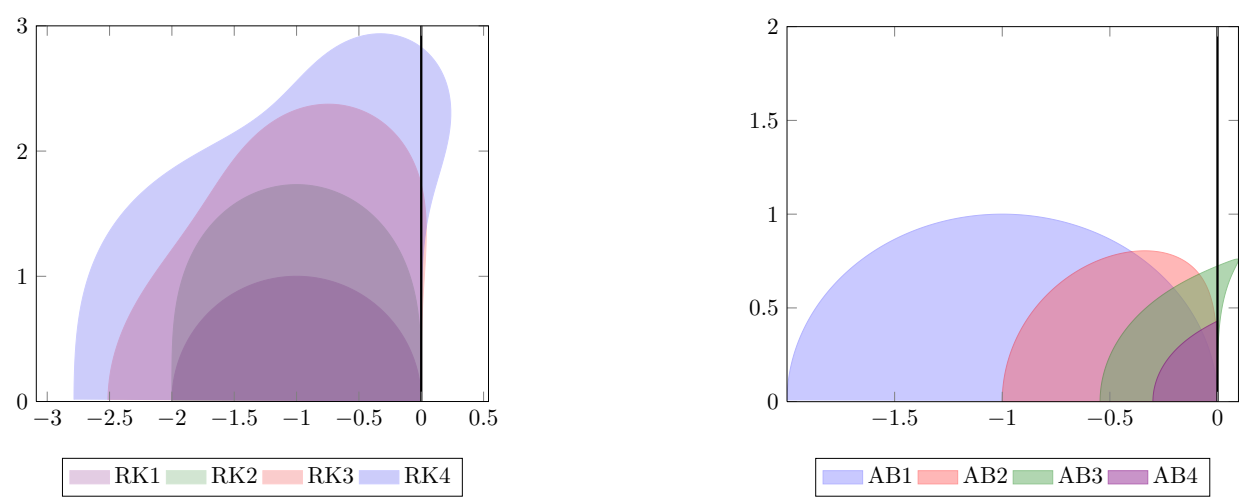

Figure 5: Von Neumann stability domains for the first four Runge-Kutta and Adams-Bashforth schemes. The operator $\mathbb{M}$ is along the $(O y)$ axis.

at the end of this section, we consider two implicit schemes: the first scheme, the Crank-Nicolson scheme, is directly inspired by [6], the second scheme is similar to the one in [9]. The Crank-Nicolson scheme and the stable time integrator require the resolution of linear systems.

Let us denote by $\left(\psi_{n}, \varphi_{n}, \omega_{n}\right)$ the approximate value at time $t=t_{n}$ and by $\delta t$ the time increment: that is $t_{n+1}=t_{n}+\delta t$. Then the unknown fields at time $t=t_{n+1}$ are obtained by solving

$$
\left\{\begin{array}{l}
\frac{\psi_{n+1}-\psi_{n}}{\delta t}=\left[\psi_{n}+\alpha \delta \psi, \varphi_{n}+\beta \delta \varphi\right]+\eta\left(J_{n}+\gamma \delta J\right)-\eta J_{c} \\
\frac{\omega_{n+1}-\omega_{n}}{\delta t}=\left[\omega_{n}+\alpha \delta \omega, \varphi_{n}+\beta \delta \varphi\right]+\left[\psi_{n}+\alpha \delta \psi, J_{n}+\beta \delta J\right]+\nu \Delta\left(\omega_{n}+\gamma \delta \omega\right) \\
\delta J=\Delta(\delta \psi) \\
\delta \omega=\Delta(\delta \varphi)
\end{array}\right.
$$

with the same initial and boundary conditions as before, and $\alpha, \beta, \gamma \in[0,1]$ parameters of the numerical scheme for the time integration. Neglecting higher order cross terms, the matricial formulation of our system of equations is

$$
\mathbb{M}\left(\begin{array}{c}
\delta \psi \\
\delta \varphi \\
\delta J \\
\delta \omega
\end{array}\right)=\delta t\left(\begin{array}{c}
{\left[\psi_{n}, \varphi_{n}\right]+\eta\left(J_{n}-J_{c}\right)} \\
{\left[\omega_{n}, \varphi_{n}\right]+\left[\psi_{n}, J_{n}\right]+\nu \Delta \omega_{n}} \\
0 \\
0
\end{array}\right)
$$

where

$$
\mathbb{M}(\cdot):=\left(\begin{array}{cccc}
I_{d}+\alpha \delta t\left[\varphi_{n}, \cdot\right] & -\beta \delta t\left[\psi_{n}, \cdot\right] & -\gamma \eta \delta t I_{d} & 0 \\
\alpha \delta t\left[J_{n}, \cdot\right] & -\beta \delta t\left[\omega_{n}, \cdot\right] & -\beta \delta t\left[\psi_{n}, \cdot\right] & I_{d}+\alpha \delta t\left[\varphi_{n}, \cdot\right]-\gamma \nu \delta t \Delta(\cdot) \\
-\Delta(\cdot) & 0 & I_{d} & 0 \\
0 & -\Delta(\cdot) & 0 & I_{d}
\end{array}\right)
$$

Remark 3.5. Only the resolution of a global linear system is needed in order to compute the fields at the new time step.

\subsubsection{Crank-Nicolson time integrator: $\alpha=\beta=\gamma=\frac{1}{2}$}

It is based on a linearised Crank-Nicolson scheme. The equation $\partial_{t} u=F(u)$ is discretized in time by the following order two scheme:

$$
\frac{u_{n+1}-u_{n}}{\delta t}=\frac{1}{2}\left(F\left(u_{n}\right)+F\left(u_{n+1}\right)\right)
$$


with $u_{n+1}=u_{n}+\delta u$, then we have to solve

$$
\underbrace{\left(I_{d}-\frac{\delta t}{2} \nabla F\left(u_{n}\right)\right)}_{\mathbb{M}} \delta u=\delta t F\left(u_{n}\right) .
$$

In the case of the reduced MHD equations (12), the system to solve writes

$$
\mathbb{M}\left(\begin{array}{c}
\delta \psi \\
\delta \varphi \\
\delta J \\
\delta \omega
\end{array}\right)=\delta t\left(\begin{array}{c}
{\left[\psi_{n}, \varphi_{n}\right]+\eta\left(J_{n}-J_{c}\right)} \\
{\left[\omega_{n}, \varphi_{n}\right]+\left[\psi_{n}, J_{n}\right]+\nu \Delta \omega_{n}} \\
0 \\
0
\end{array}\right)
$$

where the matrix $\mathbb{M}$ corresponds to

$$
\mathbb{M}(\cdot):=\left(\begin{array}{cccc}
I_{d}+\frac{\delta t}{2}\left[\varphi_{n}, \cdot\right] & -\frac{\delta t}{2}\left[\psi_{n}, \cdot\right] & -\frac{\delta t \eta}{2} I_{d} & 0 \\
\frac{\delta t}{2}\left[J_{n}, \cdot\right] & -\frac{\delta t}{2}\left[\omega_{n}, \cdot\right] & -\frac{\delta t}{2}\left[\psi_{n}, \cdot\right] & I_{d}+\frac{\delta t}{2}\left[\varphi_{n}, \cdot\right]-\frac{\delta t \nu}{2} \Delta(\cdot) \\
-\Delta(\cdot) & 0 & I_{d} & 0 \\
0 & -\Delta(\cdot) & 0 & I_{d}
\end{array}\right)
$$

Although the discrete unknowns usually satisfy $J \in \mathbb{P}^{0}, \varphi, \omega \in \mathbb{P}^{1}, \psi \in \mathbb{P}^{2}$, we tested the two cases $\psi, \varphi, J, \omega \in \mathbb{P}^{1}$ and $\psi, \varphi, J, \omega \in \mathbb{P}^{2}$ with FreeFem++. The solution has also to satisfy homogeneous Dirichlet boundary conditions for the four fields $\psi=\varphi=J=\omega=0$ on $\partial \Omega$, as well as the initial conditions $-\Delta^{*} \psi_{0}=J_{0}=J_{c}$ and $\omega_{0}=\varphi_{0}=0$ on $\Omega$ at $t=0$. In the scope of the analysis 3.1, the domain of stability of the Crank-Nicolson scheme is the half plan $\{\Re \mathrm{e}(z) \leq 0\}$. This domain has to be compared with the stability domains of explicit schemes (see Figure 5) which are bounded and which therefore have to satisfy CFL conditions. So, notwithstanding the non-linearity, the Crank-Nicolson scheme is stable for these equations in the sense that it does not have to satisfy any CFL condition $(c f .[7,10])$.

\subsubsection{Unconditional stable time integrators}

First scheme: $\alpha=0, \beta=\gamma=1$. This method is similar to the one proposed in [9, page 131]. This choice in the definition of the matrix (15) corresponds to the system

$$
\left\{\begin{array}{l}
\frac{\psi_{n+1}-\psi_{n}}{\delta t}=\left[\psi_{n}, \varphi_{n+1}\right]+\eta J_{n+1}-\eta J_{c} \\
\frac{\omega_{n+1}-\omega_{n}}{\delta t}=\left[\omega_{n}, \varphi_{n+1}\right]+\left[\psi_{n}, J_{n+1}\right]+\nu \Delta \omega_{n+1} \\
J_{n+1}=\Delta \psi_{n+1} \\
\omega_{n+1}=\Delta \varphi_{n+1}
\end{array}\right.
$$

This scheme is unconditionally stable, but first order and has the following properties.

Proposition 3.6. [Discrete Energy Conservation] Assume that $J_{c}=0$ and $\alpha=0, \beta=\gamma=1$. One has the inequality for all $\delta t>0$

$$
\int_{\Omega}\left|\nabla \psi_{n+1}\right|^{2}+\left|\nabla \varphi_{n+1}\right|^{2} \mathrm{~d} \mathbf{x} \leq \int_{\Omega}\left|\nabla \psi_{n}\right|^{2}+\left|\nabla \varphi_{n}\right|^{2} \mathrm{~d} \mathbf{x}
$$


Proof. We multiply the equation (17a) by $-\Delta \psi_{n+1}$ and the equation (17b) by $-\varphi_{n+1}$. After integration by parts using the Dirichlet boundary condition, one gets the identities

$$
\begin{gathered}
\int_{\Omega}\left|\nabla \psi_{n+1}\right|^{2} \mathrm{~d} \mathbf{x}=\int_{\Omega} \nabla \psi_{n+1} \cdot \nabla \psi_{n} \mathrm{~d} \mathbf{x}-\delta t \int_{\Omega}\left[\psi_{n}, \varphi_{n+1}\right] \Delta \psi_{n+1} \mathrm{~d} \mathbf{x}-\delta t \eta \int_{\Omega}\left|J_{n+1}\right|^{2} \mathrm{~d} \mathbf{x}, \\
\int_{\Omega}\left|\nabla \varphi_{n+1}\right|^{2} \mathrm{~d} \mathbf{x}=\int_{\Omega} \nabla \varphi_{n+1} \cdot \nabla \varphi_{n} \mathrm{~d} \mathbf{x}-\delta t \int_{\Omega}\left[\omega_{n}, \frac{\left(\varphi_{n+1}\right)^{2}}{2}\right] \mathrm{d} \mathbf{x}-\delta t \int_{\Omega}\left[\psi_{n}, \Delta \psi_{n+1}\right] \varphi_{n+1} \mathrm{~d} \mathbf{x}-\delta t \eta \int_{\Omega}\left(\omega_{n+1}\right)^{2} \mathrm{~d} \mathbf{x} .
\end{gathered}
$$

One has $\delta t \int_{\Omega}\left[\omega_{n}, \frac{\left(\varphi_{n+1}\right)^{2}}{2}\right] \mathrm{d} \mathbf{x}=0$ and

$$
\int_{\Omega}\left[\psi_{n}, \varphi_{n+1}\right] \Delta \psi_{n+1} \mathrm{~d} \mathbf{x}+\delta t \int_{\Omega}\left[\psi_{n}, \Delta \psi_{n+1}\right] \varphi_{n+1} \mathrm{~d} \mathbf{x}=0,
$$

therefore

$$
\int_{\Omega}\left|\nabla \psi_{n+1}\right|^{2} \mathrm{~d} \mathbf{x}+\int_{\Omega}\left|\nabla \varphi_{n+1}\right|^{2} \mathrm{~d} \mathbf{x} \leq \int_{\Omega} \nabla \psi_{n+1} \cdot \nabla \psi_{n} \mathrm{~d} \mathbf{x}+\int_{\Omega} \nabla \varphi_{n+1} \cdot \nabla \varphi_{n} \mathrm{~d} \mathbf{x} .
$$

The use of the Cauchy-Schwartz inequality ends the proof.

Remark 3.7. Our numerical tests show that the numerical solution captured by this time integrator has the same features that the one captured by the Cranck-Nicolson time integrator.

Second scheme: $\alpha=0, \beta=\gamma=\frac{1}{2}$. This choice in the definition of the matrix $\mathbb{M}$ corresponds to the system

$$
\left\{\begin{array}{l}
\frac{\psi_{n+1}-\psi_{n}}{\delta t}=\left[\psi_{n}, \frac{\varphi_{n+1}+\varphi_{n}}{2}\right]+\eta \frac{J_{n+1}+J_{n}}{2}-\eta J_{c}, \\
\frac{\omega_{n+1}-\omega_{n}}{\delta t}=\left[\omega_{n}, \frac{\varphi_{n+1}+\varphi_{n}}{2}\right]+\left[\psi_{n}, \frac{J_{n+1}+J_{n}}{2}\right]+\nu \Delta \frac{\omega_{n+1}+\omega_{n}}{2} \\
J_{n+1}=\Delta \psi_{n+1}, \\
\omega_{n+1}=\Delta \varphi_{n+1} .
\end{array}\right.
$$

We obtain the same property (18). The proof is almost the same as the proof of proposition 3.6: we multiply the first equation by $-\Delta\left(\frac{\psi_{n+1}+\psi_{n}}{2}\right)$ and the second one by $-\frac{\varphi_{n+1}+\varphi_{n}}{2}$ and perform the same kind of integration by parts. It is reasonable to think that this method has better preservation properties for the energy.

\subsubsection{Adams-Bashfoth time integrator}

As suggested by part 3.1, it is possible to use an explicit numerical integration in time for the non-linear terms. We have tested the Adams-Bashforth order two scheme for the non-linear terms and coupled to an implicit scheme for the diffusion. As the space discretization is of low order, the numerical viscosity is sufficient to stabilize the scheme. So this scheme turns out to be stable under the CFL stability condition $\delta t \leq C h / \mu$ with $C$ slightly inferior to $1 / 2$, and $\mu$ the sup of the derivatives of $\psi$ and $\varphi$. The numerical integration in time is given by:

$$
\left\{\begin{array}{l}
\left.\left(I d-\frac{\delta t}{2} \eta \Delta\right) \psi_{n+1}=\psi_{n}+\frac{3}{2} \delta t\left(\left[\psi_{n}, \varphi_{n}\right]-\eta J_{c}\right)-\frac{1}{2} \delta t\left(\left[\psi_{n-1}, \varphi_{n-1}\right]-\eta J_{c}\right)+\frac{\delta t}{2} \eta \Delta \psi_{n}\right), \\
\left.\left(I d-\frac{\delta t}{2} \nu \Delta\right) \omega_{n+1}=\omega_{n}+\frac{3}{2} \delta t\left(\left[\omega_{n}, \varphi_{n}\right]+\left[\psi_{n}, \Delta \psi_{n}\right]\right)-\frac{1}{2} \delta t\left(\left[\omega_{n-1}, \varphi_{n-1}\right]+\left[\psi_{n-1}, \Delta \psi_{n-1}\right]\right)+\frac{\delta t}{2} \nu \Delta \omega_{n}\right), \\
\Delta \varphi_{n+1}=\omega_{n+1} .
\end{array}\right.
$$

Contrarily to a standard explicit Euler scheme, the Adams-Bashforth scheme of order two allows to compute a solution for the Current Hole experiment on the FreeFem++ platform. The solution is converged with a smaller $\delta t$ than for the Crank-Nicolson scheme ( $c f$. Figure 6). The explicit treatment of the non-linear terms restricts the linear equations to symmetric operators and allows to speed up the computations. 


\subsection{Numerical results and discussion}

We tested three different numerical schemes to solve the Current Hole. Regarding the initial conditions, the initial magnetic flux $\psi_{0}$ is obtained by solving the Laplace equation (12c) with homogeneous boundary conditions. The initial electric potential $\varphi_{0}$ and vorticity $\omega_{0}$ are taken equal to zero. In our test the source term is $[6]$

$$
J_{c}=j_{1}\left(1-r^{4}\right)-j_{2}\left(1-r^{2}\right)^{8} \quad \text { with } j_{1}=02 \text { and } j_{2}=0.2666 \text {. }
$$

The viscosity is $\nu=10^{-6}$ and the resistivity $\eta=10^{-5}$. In Figure 6 , we represent the final results at $t=2000$ of numerical experiments with the Crank-Nicolson scheme and with the Adams-Bashforth second order scheme. We also compare with the unconditionally stable algorithm. The results are visually very similar. The error is totally dominated by the space discretization.

Using an explicit scheme (such as the second order Adams-Bashfoth scheme) allows to diminish the CPU burden per iteration for the computation of the solution of the next step (16). In a Galerkin formulation, there is no numerical viscosity coming from the space discretization to stabilize the numerical scheme. Hence, the Adams-Bashforth scheme order two, which enhances the small scales (see the graph of stability Fig. 5 right) may create spurious oscillations. Therefore, it requires the stronger stability condition $\Delta t \leq C h^{\frac{4}{3}}$ than the CFL condition (see [7] for more details). As a result, it needs much smaller time steps than the Crank-Nicolson scheme, whose linear stability domain is exactly the left half-plan (to compare with Figure 5) and which does not have to satisfy any CFL condition in order to be stable. However, for large time steps, the Crank-Nicolson scheme becomes unstable because the equations (12a)-(12d) are non linear. The unconditionally stable algorithm is stable for even larger time steps (up to $\delta t=10$ ).

In an attempt to familiarize the reader with the FreeFem++ platform, we briefly describe essential steps for coding the Crank-Nicolson scheme. Once the domain mesh is constructed and the appropriate $\mathbb{P}^{1}$ or $\mathbb{P}^{2}$ finite element spaces are initialized, we are ready to define the components of the matrix $\mathbb{M}$ and of the right hand side vector. The following piece of FreeFem++ script illustrates the implementation: one should first define the variational form for each matrix component, and then to build the global matrix.

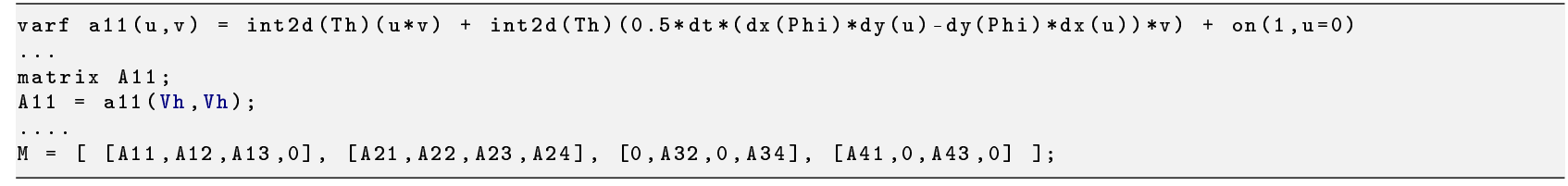

The matrix is non symmetric. When choosing a right solver, it is important to consider hardware resources, mainly available memory. For relatively coarse discretizations, we advocate a use of the direct UMFPACK solver, based on the LU factorization. For fine meshes, iterative solver, such as GMRES could be used. However, the authors observed difficulties in convergence of a GMRES solver. The reasons for this poor performance are subject to further investigations.

set $(M$, solver $=$ UMFPACK, eps $=1 e-8)$

In the case of the Adams-Bashforth scheme order two, the convective terms are computed explicitly so the matrix (16) is symmetric, positive-definite and invariant along the time. This allows to use a conjugate gradient solver (GMRES) or to invert the matrix (16) at the beginning of the routine once for all the time steps.

The code has been run on a cluster node with two Intel Xeon E5540 processors, i.e. 8 cores in total. For this particular example, the spatial resolution has been chosen $h=0.01$ for $\Omega=\mathcal{C}(0,1)$, which leads to approximately $142 \cdot 10^{3}$ degrees of freedom when using $\mathbb{P}^{1}$ elements. It takes 0.2 second per time step when using a parallel version of the direct solver, UMFPACK V5.4, INTEL MKL V10.3.

Our results for the current hole problem are comparable to the ones obtained in [6]. The profile exhibits a zone of negative current density close to the axis namely the "current hole". Figure 7 shows snapshots of the current profile at different stages of the internal kink evolution. The current surface, initially axisymmetric, remains identical during the linear stage in the early part of the transient, where non-linear effects are still negligible. At the end of this stage, the profile is progressively deformed: current density is expelled outward 


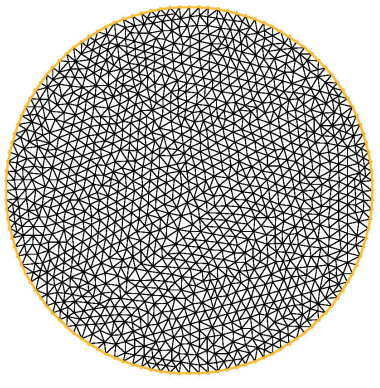

(a) Mesh, $h \sim 0.05$

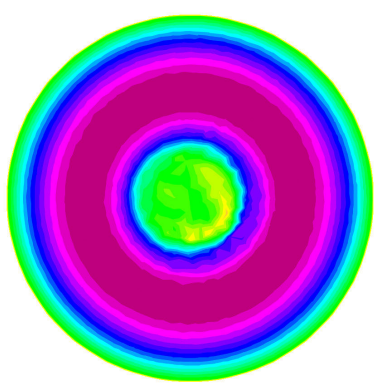

(c) Adams-Bashforth order 2, $\delta t=0.1$

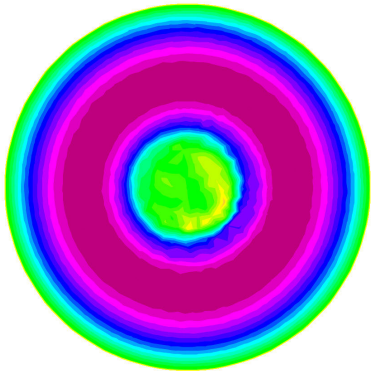

(b) Crank-Nicolson, $\delta t=1$

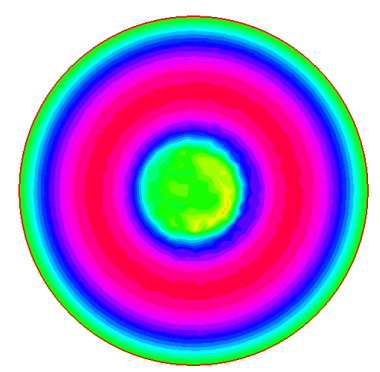

(d) Unconditionally stable scheme, $\delta t=2$

Figure 6: Numerical stability of different time integrator, $t=2000$. All three results are very similar. This probably is due to the fact that the mesh is the same. The time step is greater for the unconditionally stable scheme.

from the central axis, generating a current sheet at the resonant surface. At the end of the crash, the profile close to the centre is flattened, leaving only residual fluctuations around $J=0$. Finally we notice that the original problem is invariant by rotation: therefore the orientation and dynamics of the instability can be triggered by the mesh used for the simulation and by the time step; in particular a similar instability is observed in Figure 6 and in Figure 7, but at different angle and time.

\section{Conclusion and Perspectives}

We have presented the use of the FreeFem++ computational tool for the numerical solution of resistive ideal MHD equations adapted to realistic geometrical tokamak configurations. The method was first applied for the computation of the equilibrium problem and then to the non stationary problem. Some conclusions can already be established for the equilibrium problem: FreeFem++ is very easy to handle and adapted to solve this kind of problems in general geometry, we observe a good agreement with the literature; some questions remain open. It is essentially related to polynomial non-linear right hand sides: in the case of multiple solutions, we need a criteria to select the physical one; is there a criterion to decide a priori what is the more adapted Finite Element type. Concerning the application to the computation of the time-dependent problem of a current hole in a cylindrical geometry, the results are consistent with the results [6] that were obtained with a completely different method. Since the mesh is a general unstructured one, the mesh is by itself a source of perturbation at time $t=0$. In consequence there is no need of an initial perturbation for the numerical characterization of 


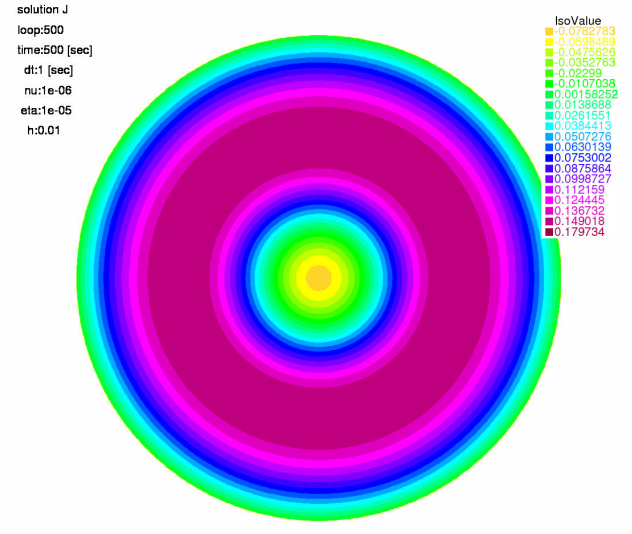

(a) $t=500$

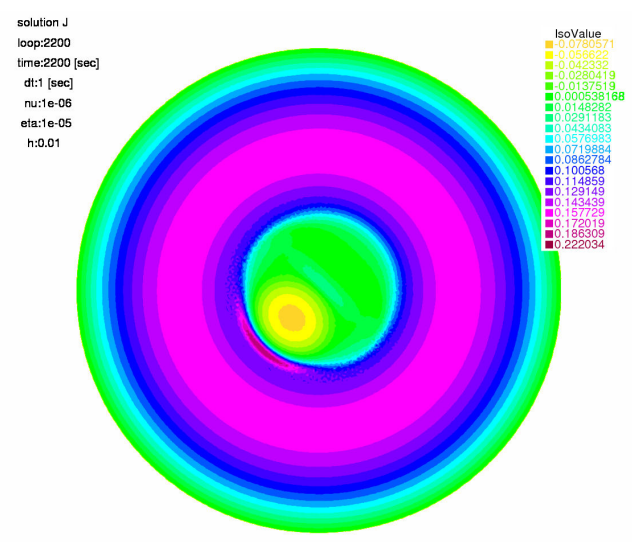

(c) $t=2200$

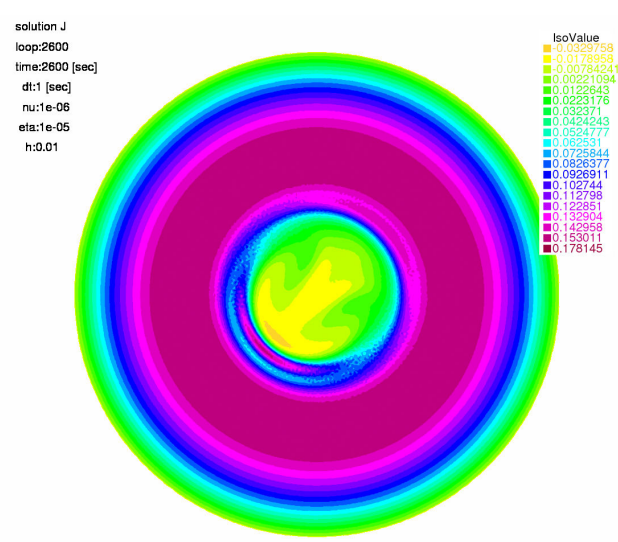

(e) $t=2600$

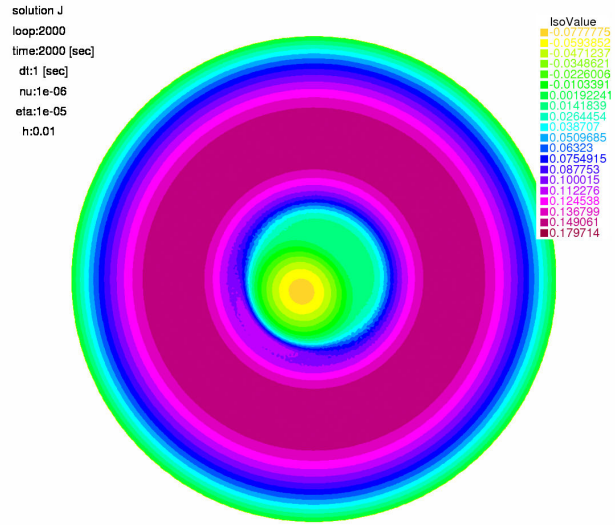

(b) $t=2000$

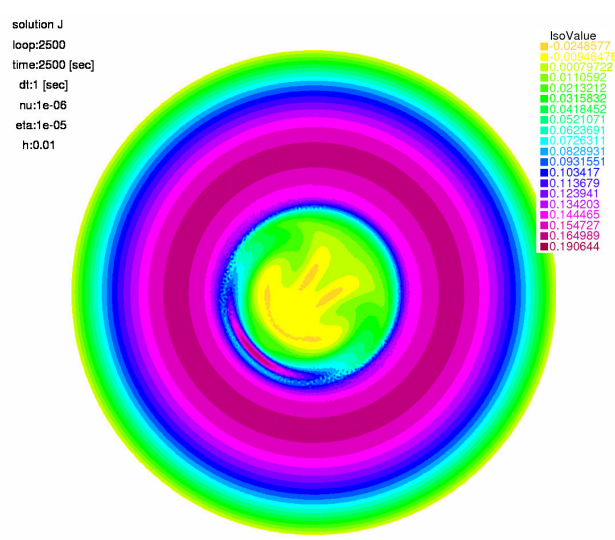

(d) $t=2500$

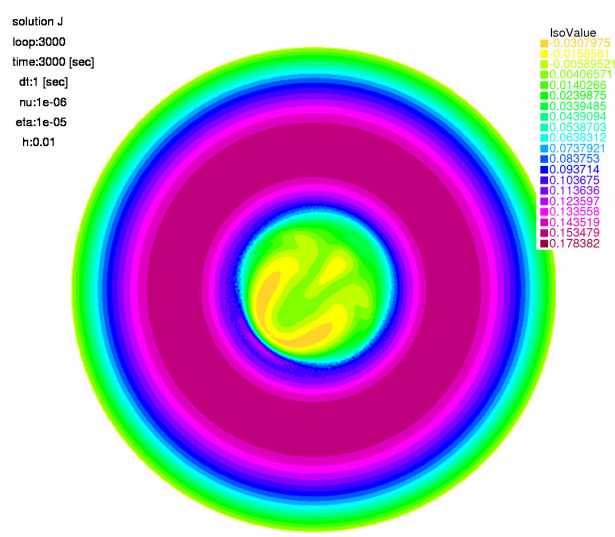

(f) $t=3000$

Figure 7: Plots of the current $J$ computed with FreeFem++. Numerical experiment for the Current Hole, equations (12) discretized with $\mathbb{P}^{1}$ finite elements and Crank-Nicolson scheme in time with $h=0.01, \delta t=1$. We observe that the initial stationary solution is unstable. 
the current hole. It is possible to evaluate easily different time integrators on the same problem (FreeFem++ scripts used in this paper can be downloaded at http://www.ann.jussieu.fr/ gostaf/emaff/).

A general conclusion is that FreeFem++ is very well adapted for the effective simulation of such non trivial plasma problems.

Acknowledgement. We warmly thank Guido Huysmans for useful discussions and technical contributions at the CEMRACS'10. This project was founded by the AEN Fusion of INRIA.

\section{REFERENCES}

[1] 2nd UN Conf. on the Peaceful Uses of Atomic Energy. Hydromagnetic Equilibria and Force-Free Fields, volume 31, 1958.

[2] S.H. Aydin and M. Tezer-Sezgin. Numerical solution of grad-shafranov equation for the distribution of magnetic flux in nuclear fusion devices. Turkish Journal of Engineering \& Environmental Sciences, 32(5):265-275, 2008.

[3] D. Biskamp. Nonlinear magnetohydrodynamics. Cambridge Univ Pr, 1997.

[4] S. Briguglio, G. Wad, F. Zonca, and C. Kar. Hybrid magnetohydrodynamic-gyrokinetic simulation of toroidal alfven modes. Phys. Plasmas, 2 (10):3711-3723, 1995.

[5] S. Briguglio, F. Zonca, and C. Kar. Hybrid magnetohydrodynamic-particle simulation of linear and nonlinear evolution of alfven modes in tokamaks. Phys. Plasmas, 5 (9):3287-3301, 1998.

[6] O. Czarny and G. Huysmans. Bézier surfaces and finite elements for mhd simulations. Journal of Computational Physics, 227(16):7423-7445, 2008.

[7] E. Deriaz and D. Kolomenskiy. Stability of explicit numerical schemes for convection-dominated problems. arXiv:1007.3313v1.

[8] J.P. Freidberg. Plasma physics and fusion energy. Cambridge Univ Pr, 2007.

[9] J.F. Gerbeau, C. Le Bris, and T. Lelièvre. Mathematical methods for the magnetohydrodynamics of liquid metals. Oxford University Press, USA, 2006.

[10] E. Godlewski and P.-A. Raviart. Hyperbolic Systems of Conservation Laws, volume 3/4 of Mathématiques 83 Applications (Paris) [Mathematics and Applications]. Ellipses, Paris, 1991.

[11] F. Hecht, O. Pironneau, A. Le Hyaric, and K. Ohtsuka. Freefem ++ Manual, 2010.

[12] A. H. Khater and S. M. Moawad. Exact solutions for axisymmetric nonlinear magnetohydrodynamic equilibria of aligned magnetic field and plasma flow with applications to astrophysics and plasma confinement devices. Physics of Plasmas, 16, 2009.

[13] V.D. Shafranov. Plasma equilibrium in a magnetic field. Reviews of Plasma Physics, 2:103, 1966.

\section{CoDe For THE CURRENT HOLE: THE CRAnK-NiCOLSOn SCHEME}

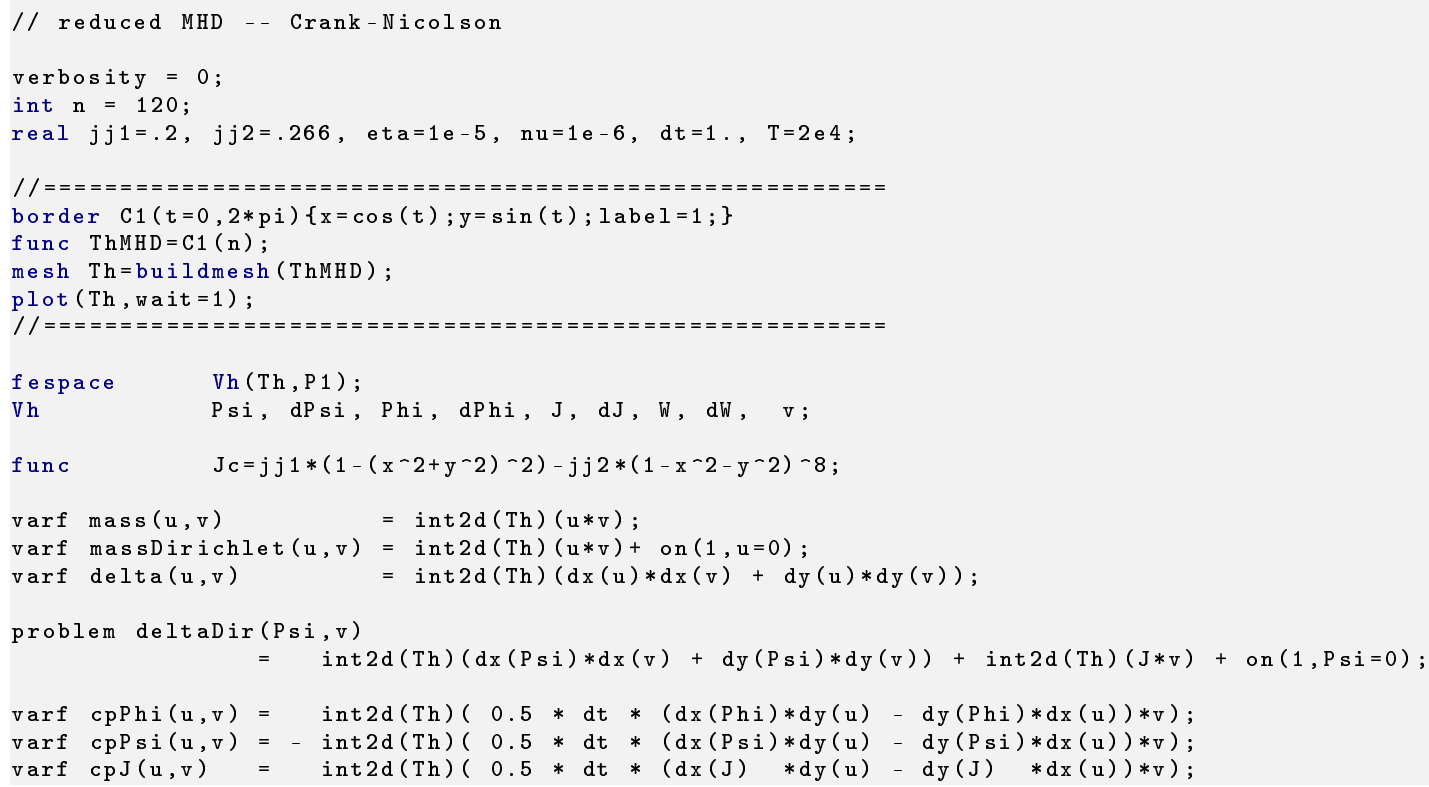




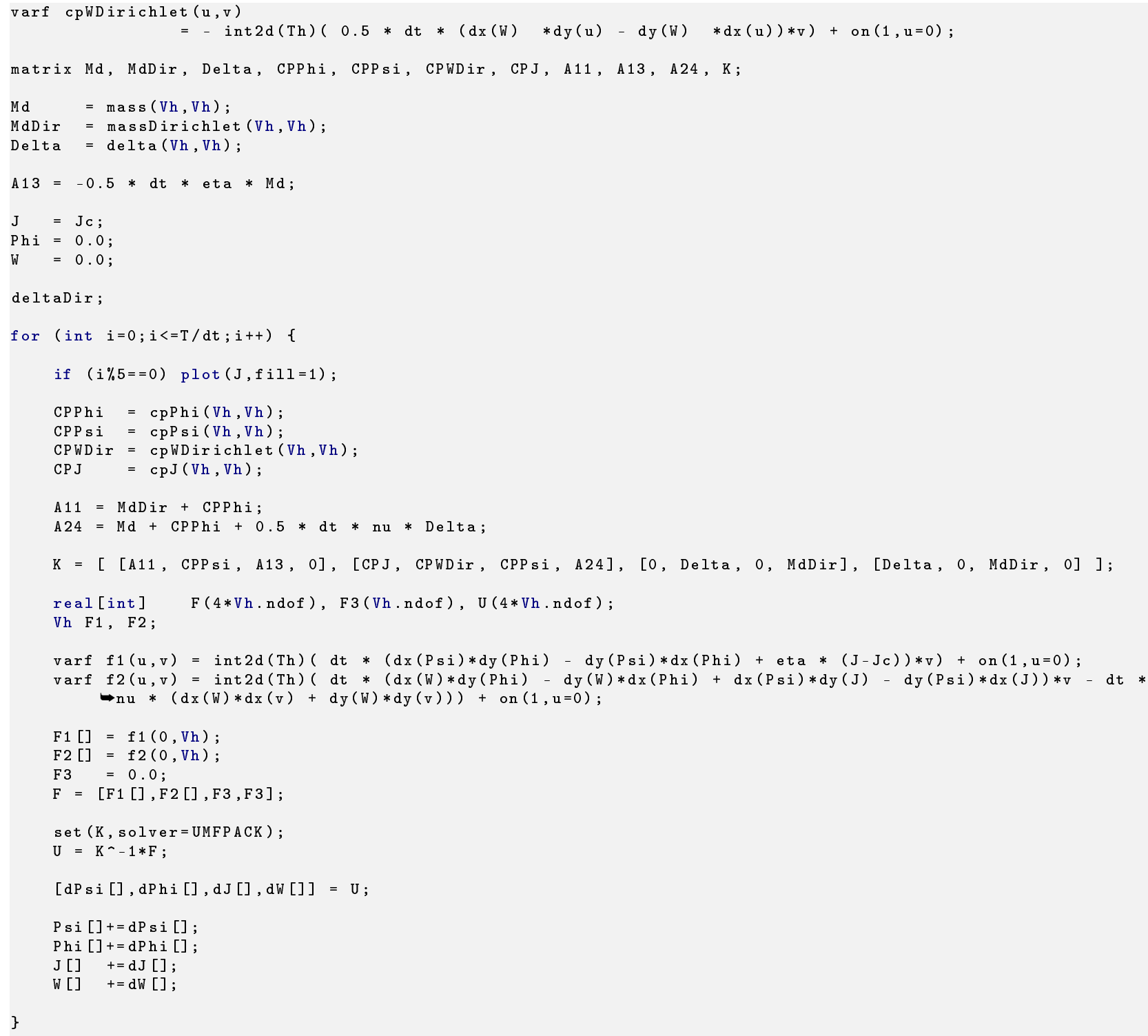

